\title{
Prolonged survival after portal decompression of patients with non-cirrhotic intrahepatic portal hypertension
}

\author{
R. ZEEGEN ${ }^{1}$, A. G. STANSFELD, A. M. DAWSON, AND A. H. HUNT² \\ From St Bartholomew's Hospital, London
}

SUMMARY In a series of 251 good-risk patients undergoing portal decompression for intra- $\frac{\rho}{\circ}$ hepatic portal hypertension, one fifth have been found not to have hepatic cirrhosis. Of these,응 44 had only minor changes in hepatic architecture, and the clinical features and subsequent $\rightarrow$ course have been compared and contrasted with a group of 201 cirrhotic patients who under- $\subseteq$ went portal decompression for similar indications.

The degree of portal hypertension was comparable in both groups and it was not possible $\vec{\theta}$ confidently to differentiate the condition from hepatic cirrhosis on either clinical or bio-. chemical grounds in the individual case.

The histological lesion was not progressive in the non-cirrhotic group and this was reflected in the far better survival of these patients. After five years $83 \%$ (30 of 36) of this group were alive compared with $43 \%$ (65 of 152) of the cirrhotic patients. After 10 years the cumulative $\frac{\mathscr{Q}}{\mathbb{D}}$

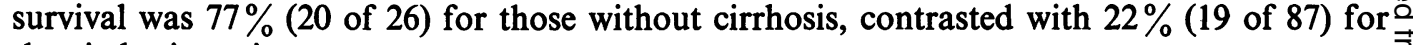
the cirrhotic patients.

Portal hypertension in the absence of nodular cirrhosis or extrahepatic portal venous obstruction is a recognized but uncommon clinical entity. Amongst the established causes are congenital hepatic fibrosis (Kerr, Harrison, Sherlock, and Walker, 1961), partial nodular transformation (Sherlock, Feldman, Moran, and Scheuer, 1966), hepatic granulomata (Porter, 1961), primary biliary cirrhosis (Zeegen, Stansfeld, Dawson, and Hunt, 1969), alcoholic liver disease (Reynolds, Hidemura, Michel, and Peters, 1969), 'tropical splenomegaly' (Leather, 1961 ; Williams, Parsonson, Somers, and Hamilton, 1966), and the myeloproliferative syndrome (Shaldon and Sherlock, 1962). In addition to these specific disorders, scattered reports have appeared from both tropical and temperate areas of patients with intrahepatic portal hypertension without cirrhosis, but with a variety of less well defined hepatic abnormalities. In some instances the liver was considered to be histologically normal (Polish, Christie, Cohen, and Sullivan, 1962; Miller and Brandt, 1962), but in the majority of cases mild portal fibrosis - was present either alone (Siderys and Vellios, 1964) or in combination with sinusoidal dilatation (Tisdale, Klatskin, and Glenn, Received for publication 31 December 1969.

'Present address: Westminster Hospital, London, SW1 'Mr Hunt died on 4 July 1970.
1959) and cellular infiltration (Ramalingaswami, Wig, and Sama, 1962; Imanaga, Yamamoto, and $\stackrel{\circ}{=}$ Kuroyanagi, 1962). In addition vascular and lobular abnormalities were described, including $\stackrel{3}{3}$. thickened portal vein radicles, focal nodulation, $\delta$ and more subtle disturbances of hepatic archi- $₹$ tecture (Mikkelsen, Edmonson, Peters, Redeker,은 and Reynolds, 1965; Basu Mallik, Sen Gupta, $>$ Basu, Biswas, Pal, and Boyer, 1967; Boyer, Sen Gupta, Biswas, Pal, Basu Mallik, Iber, and Basu, N 1967; Nayak and Ramalingaswami, 1969).

We now report a further 44 similar cases in $\tilde{O}$ which portal decompression was carried out for $N^{N}$ gastrointestinal haemorrhage where the portalo vein was patent and the liver was not cirrhotic.co Two hundred and one patients with hepatic $\widetilde{\Phi}$ cirrhosis of mixed aetiology underwent portal de-? compression during a similar period and with ${ }^{\circ}$ similar criteria for operation, and so the oppor-웅 tunity was taken to compare and contrast the $\stackrel{\square}{\circ}$ clinical, biochemical, and prognostic features of $\stackrel{\mathbb{\Phi}}{\propto}$ the two groups.

\section{Material and Methods}

A systematic retrospective study was undertaken 
of the liver histology and clinical features of 249 patients who had undergone portal decompression for intrahepatic portal hypertension between 1948 and 1967. Adequate operative liver biopsies were available from all but three cases and necropsy material or information was obtained in 92 cases. Reticulin and haematoxylin and eosin preparations were studied and initially 51 biopsies emerged in which the histological criteria of cirrhosis appeared to be absent. Reference was then made to the case histories, paying particular attention to the macroscopic appearance of the liver and the state of the extrahepatic venous system. If the surgeon described the liver as grossly or uniformly nodular, or if it appeared that the primary site of the portal hypertension was, in retrospect, extrahepatic portal obstruction, the case was excluded. At necropsy if the liver showed nodular cirrhosis the case was excluded. In fact, this system led to the exclusion of only three cases from the non-cirrhotic group (one case each of macronodular cirrhosis, secondary biliary cirrhosis, and probable schistosomal cirrhosis) leaving a total of 48 cases. Of these, four patients had congenital hepatic fibrosis and two had extensive hepatic granulomata of uncertain aetiology. There then remained 42 $(16.9 \%)$ cases with apparently minor histological changes in the liver. A further two patients, who were operated on in 1969 but omitted from the survival analysis, were added for completeness. These 44 patients formed the basis of this study (Table I).

Thirty-five of these patients were decompressed by portacaval and nine by splenorenal anastomosis. In all but one of the former group and five of the latter the operation was the standard end-to-side procedure (Hunt, 1958). In four cases splenectomy had been carried out one month, eight, 13, and 17 years before decompression. In two cases the shunt was preceded by transthoracic oesophagotomy and ligation of varices (Crile, 1953) for the emergency control of haemorrhage.

Routine preoperative blood counts and serum bilirubin and serum albumin estimations were carried out in most cases. A bilirubin level generally below 2 to $3 \mathrm{~g} / 100 \mathrm{ml}$ and albumin level of at least $3.0 \mathrm{~g} / 100 \mathrm{ml}$ were prerequisites before

\begin{tabular}{lclr}
\hline Non-cirrhotic & $\begin{array}{l}\text { No. of } \\
\text { Patients }\end{array}$ & Cirrhotic & $\begin{array}{l}\text { No. of } \\
\text { Patients }\end{array}$ \\
\hline Portal and parenchymal & & Cryptogenic & 114 \\
changes & $44^{1}$ & Alcoholic & 35 \\
Congenital hepatic fibrosis & 4 & Biliary (primary and secondary) & 30 \\
Granulomata & 2 & Chronic active hepatitis & 8 \\
& 50 & Others $^{2}$ & 14 \\
Total & 50 & & 201 \\
\hline
\end{tabular}

Table I Classification of 251 patients with intrahepatic portal hypertension

IIncluding two patients operated on in 1969.

'Including haemochromatosis, Wilson's disease, and schistosomal cirrhosis. operation. Alkaline phosphatase was estimated in 34 cases, serum aspartate amino transferase (SGOT) in 21 cases, and bromsulphthalein (BSP) retention at 45 minutes in 10 cases. A percutaneous splenic venogram was obtained im- $\stackrel{-}{\Rightarrow}$ mediately before operation and a portal veno- $\stackrel{5}{\stackrel{5}{9}}$ gram was taken at operation via a jejunal venous radicle after measurement of the portal pressure with a citrate manometer from the same site (Hunt, 1958).

Follow-up data were available in all 44 patients and liver function tests were also available in a number of cases at varying intervals after operation.

The results of the parallel study of 201 cirrhotic patients with portal hypertension who underwent portal decompression for comparable indications during the same period were available for comparison with the non-cirrhotic group. Follow-up data were obtained in all but one cirrhotic patient.

\section{The Liver}

\section{OPERATION}

\section{Macroscopic findings}

In the majority of cases the liver was only slightly enlarged, and the surface was described as mildly granular, irregular, or showing 'early cirrhosis'. In four cases it was smooth and covered with a fine fibrous network and in a similar number it was described as shrunken and fibrotic with a wrinkled surface. In every case the liver was firmer than normal and sutures put in at the biopsy site did not cut out.

\section{Microscopic findings}

A generous wedge biopsy was taken and it would appear that this amount was necessary to appreciate the often subtle changes in liver 을 architecture which characterized the condition. The changes will be fully described elsewhere (Stansfeld and Zeegen), but in essence they comprised minor portal tract abnormalities together with a subtle distortion of lobular architecture (Fig. 1). Attention was drawn to this by the distribution of hepatic veins which were $\square$ unduly numerous in one area and deficient in 0 another. Sometimes they were dilated and situated close up against a portal tract. The portal tract changes were characterized by a curious vascular proliferation together with a mild stellate fibrosis, often with thin septa radiating for varying distances into the parenchyma. Occasionally two or three portal tracts were linked together by these septa. Focal proliferations of liver cells could be seen and rarely these would constitute a true nodule, particularly near the surface of the liver. However, this nodular change apparently remained entirely focal and did not become generalized even after many years. Overt liver 


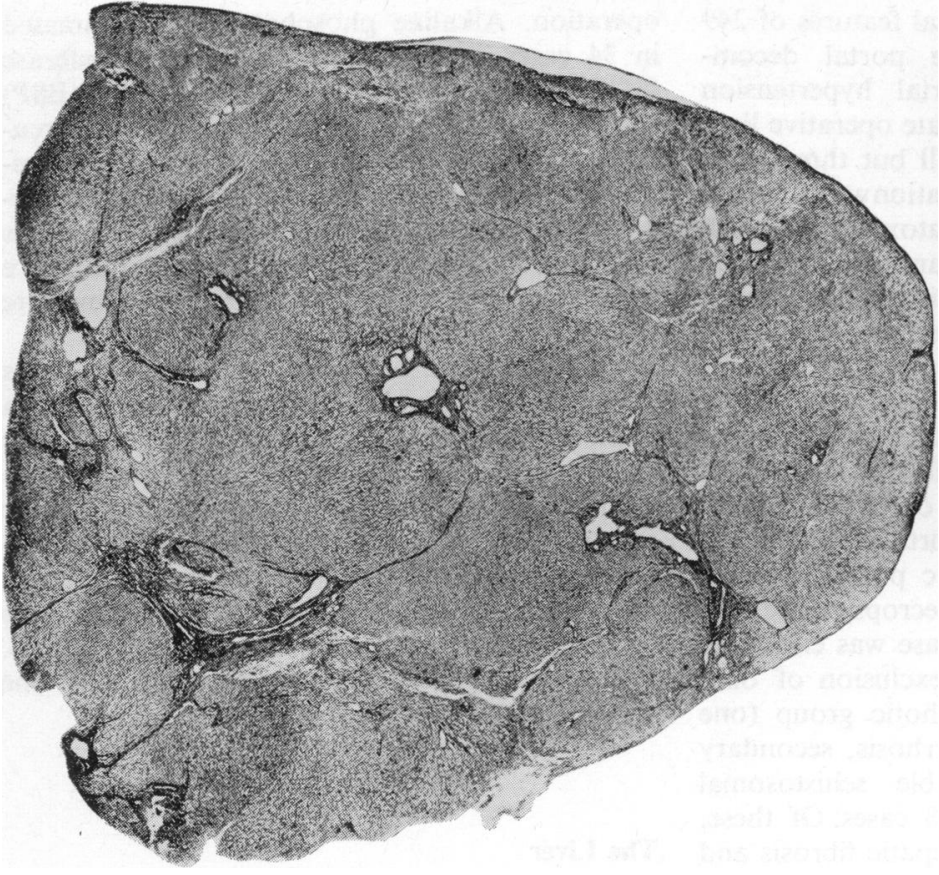

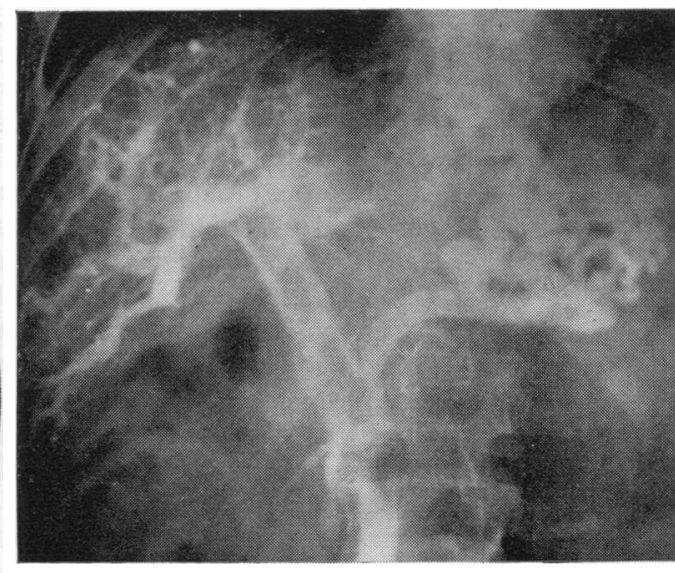

Fig. 2 Operative jejunal venogram showing an abnormal intrahepatic pattern from a patient with non-cirrhotic portal hypertension. The apparent filling defect in the portal vein was due to streaming of contrast medium. (No thrombosis was found on opening the vein.)

Fig. 1 Operative wedge biopsy from a patient with non-cirrhotic intrahepatic portal hypertension. Note the irregular distribution of hepatic veins and mild portal fibrosis (reticulin preparation $\times 9$ ).

cell necrosis was absent, as was bile ductular proliferation, marked cellular infiltration, and intimal thickening of portal venous radicles.

\section{NECROPSY}

\section{Macroscopic findings}

Eight of the 13 patients who died came to necropsy from two to 15 years after surgery. The livers were shrunken and firm apart from two which were enlarged and infiltrated with metastatic bronchial carcinoma. The cut surface was described in one case as having a nodular appearance and in another as showing a fine diffuse fibrosis.

\section{Microscopic findings}

The striking feature was the minimal change that had occurred even after many years. Fibrosis was slightly increased in a number of cases, and in four specimens intimal thickening of larger intrahepatic portal veins was apparent (Stansfeld and Zeegen, in preparation).

Thus there was no macroscopic or microscopic evidence of progression to cirrhosis in these necropsy specimens.

\section{The Portal Vein}

\section{OPERATION}

\section{Macroscopic findings} normal in 34 cases, and in four of these a patent umbilical vein was present. Abnormalities of the extrahepatic venous system in the remaining 10 patients $(22.7 \%)$ included partial thrombosis, calcification, and eccentric and concentric fibrous thickening described in some cases as 'phlebosclerosis'. In those cases where partial thrombosis was found the clot was often adherent, tough, and fibrous and needed to be scraped out with a Volkmann spoon before the porta caval anastomosis could be fashioned. Similar changes, however, were found in the portal vein in $29(14.4 \%)$ of the cirrhotic group.

\section{Venography}

Twenty-eight of the operative portal venograms were available for review. In general the intrahepatic pattern was distorted (Fig. 2) resembling the findings in hepatic cirrhosis, but in six cases the pattern appeared within normal limits with good peripheral filling of small vessels. In one case there was a curious pooling of contrast medium throughout the liver and in three cases there was insufficient contrast medium to opacify the liver as most of the dye was diverted through enormous collaterals.

\section{Portal pressure}

Both groups of patients had a comparably high portal venous pressure as estimated at operation
At operation the portal vein was fully patent and 
by direct measurement (see Methods). The mean normal value for this method was $100 \mathrm{~mm}$ water (Hunt, 1958) and the value for the non-cirrhotic patients was $302 \cdot 2 \mathrm{~mm}$ water (range 140-590) which was not significantly different from the mean value for the cirrhotic patients (Fig. 3) of $286 \cdot 6 \mathrm{~mm}$ water (range 120-600).

\section{Clinical, Haematological, and Biochemical Features}

Approximately $90 \%$ of both groups presented with gastrointestinal haemorrhage although the mean frequency of bleeds in the non-cirrhotic group before referral was $2.5(\mathrm{SD}=1.6)$ which was significantly greater than the mean value for the cirrhotic patients of $1.6(\mathrm{SD}=1.3, \mathrm{P}<0.001)$. In the remaining patients varices were demonstrated and operation was 'prophylactic'.

Of the non-cirrhotic patients, 41 were born in England. Men were more frequently affected than women: the group comprised 33 men $(75 \%)$ and 11 women $(25 \%)$. A similar male preponderance was observed in the cirrhotic group in which there were 113 men and 88 women. The non-cirrhotic men were significantly younger than those with cirrhosis but the ages of the women were similar (Table II).

In two thirds of the non-cirrhotic group there were no obvious underlying aetiological factors. Eight patients gave a history of previous jaundice and three took alcohol to excess. Four patients gave histories of prolonged ingestion of medicines containing arsenic for epilepsy and skin conditions, and all four had cutaneous evidence of chronic arsenic intoxication with pigmentation, hyperkeratosis of palms and soles, and malignant skin lesions.

Although splenomegaly was prominent in the non-cirrhotic patients, the spleen was impalpable in eight, of whom four had had a previous splenectomy. Hepatic enlargement was not marked and only in seven cases was the liver palpable to three fingerbreadths or greater. Ten

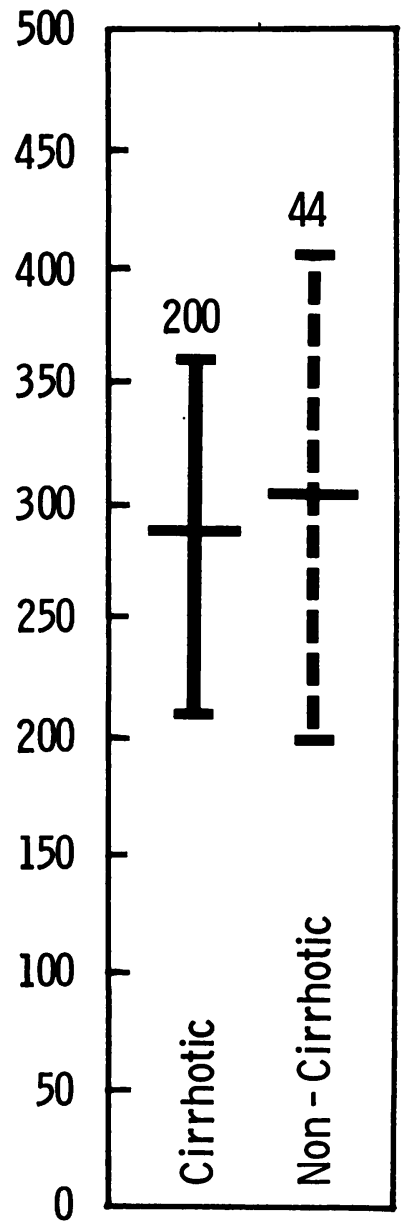

Fig. 3 Mean operative portal venous pressure in patients with intrahepatic portal hypertension $( \pm 1 S D)$. Number of patients measured given above each group.

Means $286.6 \mathrm{mmH}_{2} \mathrm{O}$ (cirrhotic); $302 \cdot 2 \mathrm{mmH}_{2} \mathrm{O}$ (non-cirrhotic)

SD 80.9 (cirrhotic); $104 \cdot 4$ (non-cirrhotic)

$P$ was not significant.

Table II Clinical, biochemical, and haematological findings in patient with intrahepatic portal hypertension 


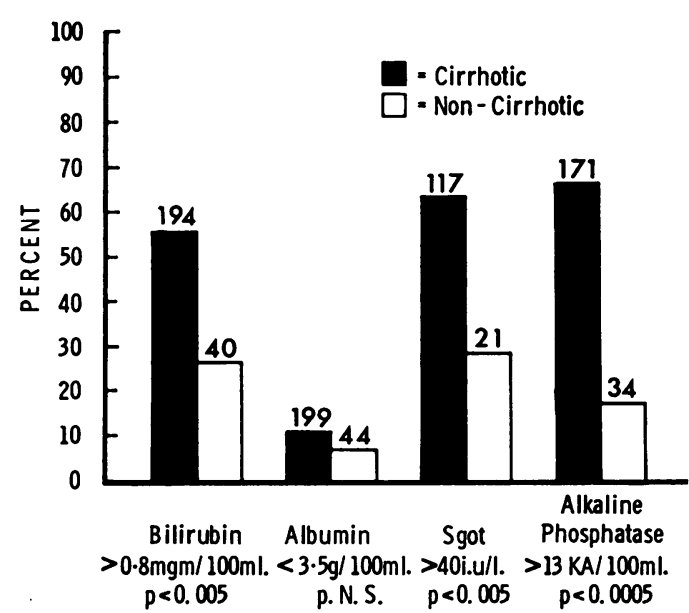

Fig. 4 Percentage of patients with intrahepatic portal hypertension with abnormal liver function at the time of portal decompression. Figures above columns denote numbers of patients in each group tested.

patients $(22 \cdot 7 \%)$ had the cutaneous stigmata associated with liver disease such as spider naevi and palmar erythema. Eleven patients $(25 \%)$ had ascites at operation or within three months prior to operation and in nine cases the ascites had developed following variceal haemorrhage.

There were no significant differences in the haematological findings between the cirrhotic and non-cirrhotic patients; these are summarized in Table II. The mean preoperative serum albumin level of $4 \cdot 6 \mathrm{~g} / 100 \mathrm{ml}$ (range $3 \cdot 1$ to $5 \cdot 7$ ) was significantly higher than that of the cirrhotic patients of $4.2 \mathrm{~g} / 100 \mathrm{ml}(\mathrm{P}<0.001)$. Nevertheless the great majority of readings from both groups were within the normal range. The remaining liver function tests were significantly better in the non-cirrhotic than in the cirrhotic. Whereas the mean serum bilirubin and alkaline phosphatase levels of the former group were normal the mean BSP value was slightly elevated (Table II). Nevertheless, the range of readings was such that in the individual case it was not possible to distinguish the cases from hepatic cirrhosis either biochemically or clinically.

The comparison between the percentage of patients with abnormal liver function tests at the time of operation are shown in Figure 4. Repeated determinations of liver function at varying periods following decompression showed an overall deterioration in the non-cirrhotic patients.

\section{Subsequent Course}

There were no operative deaths in the noncirrhotic group: the overall operative mortality for the cirrhotic patients was $10 \%$. The latter figure does, however, include a few poor-risk patients operated on early in the series, and considering the period from 1957 to 1967 the mortality was $4.3 \%$. In the non-cirrhotic patients thrombosis of the stoma occurred in two patients after decompression by splenorenal anastomosis. A proximal gastric resection and porta caval of anastomosis were carried out in these cases, one $\bar{c}$ and four years respectively after the initial operation. In the remaining 42 cases satisfactory decompression was obtained and there were no further bleeding episodes from varices.

Operation relieved ascites in all cases, and there was no reaccumulation after operation in the non-cirrhotic patients. Seven $(18.2 \%)$ of the noncirrhotic and $39(19.4 \%)$ of the cirrhotic patients developed marked portal systemic encephalopathy. The mean age of the non-cirrhotic patients with overt encephalopathy was 48.8 years $(\mathrm{SD}=13.2)$ which was significantly greater than those without encephalopathy $(36.9$ years, $S D=\overrightarrow{0}$ $11.9, \mathrm{P}<0.025)$. The respective values for the 의 cirrhotic patients did not reach any statistical $\vec{c}$ significance: the mean age of those with overt $\subseteq$ encephalopathy was 51.5 years $(S D=10.4)$ as compared with $46 \cdot 7$ years $(\mathrm{SD}=14.8, \mathrm{P}=<0 \cdot 1)$ for those without.

Furthermore, on the basis of sequential quantified psychometric testing (Zeegen, Drinkwater, and Dawson, 1970), both cirrhotic and noncirrhotic patients have been shown to have a considerable degree of cerebral dysfunction, $\stackrel{\mathbb{Q}}{\complement}$ whether clinical encephalopathy was present or $\overrightarrow{\bar{B}}$ not.

Six of the seven non-cirrhotic patients who had $\frac{\partial}{\partial}$ developed encephalopathy are still alive, and are stabilized on protein restriction alone or with lactulose (Zeegen, Drinkwater, Fenton, Vince, and Dawson, 1970) or neomycin. Two patients have a non-progressive spastic paraparesis, which is presumably vascular and one of these developed deep coma on two occasions following haemorrhage from a duodenal ulcer. 온 He remains relatively well and free from en- $\rightarrow$ cephalopathy more than 11 years after portal decompression. The only death in this group $\stackrel{N}{\circ}$ followed three months after a colonic exclusion operation for intractable encephalopathy 10 years after the shunt.

Duodenal ulcers occurred equally after portal decompression in both non-cirrhotics and cir- $\bullet$ rhotics. Six $(13.6 \%)$ of the former group and $16 \Phi$ $(8 \%)$ of the latter developed duodenal ulcer. Two of the non-cirrhotic patients subsequently $\frac{T}{0}$ bled from the ulcer: one died and the other $\stackrel{\mathbb{D}}{\stackrel{D}{0}}$

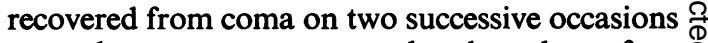
to undergo a vagotomy and pyloroplasty four $\varrho$ and a half years after portal decompression. One cirrhotic and one non-cirrhotic patient deve- $\delta$ loped overt multiple pulmonary arteriovenous fistulae with dyspnoea, cyanosis, and clubbing. Hepatoma developed in $12(6 \%)$ of the cirrhotic patients: malignant change did not occur in the non-cirrhotic group. 


\section{Prognosis}

The long-term survival of the non-cirrhotic patients was far better than that of the cirrhotics, and after five years $83 \%$ of the former were alive compared with $43 \%$ of the latter. After 10 years the cumulative survival was $77 \%$ for the noncirrhotic group compared with $22 \%$ for the patients with hepatic cirrhosis, and after 15 years only $8 \%$ of the cirrhotic group remained alive in contrast to $58 \%$ in the non-cirrhotic group.

Of the 13 non-cirrhotic patients who died only four $(9.1 \%)$ died of liver failure. This was contrasted with at least $60(30 \%)$ of the 136 cirrhotic patients who died of liver failure. Of the remainder, four died from cerebrovascular accidents, two from carcinomatosis from a bronchial tumour (both patients had suffered chronic arsenic poisoning), two from gastrointestinal haemorrhage (one from a duodenal ulcer, and one from an anastomotic ulcer at the site of a proximal gastric resection), and one patient died from chronic myeloid leukaemia.

\section{Discussion}

The surprisingly large number of patients with non-cirrhotic portal hypertension undoubtedly reflects the fact that this centre deals with a highly selected population of good-risk patients. It is well documented that the first variceal haemorrhage in a cirrhotic patient is often fatal. The reported mortality varies from $33 \%$ (Sherlock, 1964) to $86 \%$ (Cohn and Blaisdell, 1958), and many cirrhotics may die from liver failure, intercurrent infection, or hepatoma without ever having bled. In a recent retrospective survey of 155 unselected cases of cirrhosis in England, Stone, Islam, and Paton (1968) reported a fiveyear survival of $14.3 \%$ although only 31 patients $(20 \%)$ had bled. No more than six of these patients were suitable for surgery and only one patient survived porta caval anastomosis. The majority of the 251 patients included in the present study had been transferred to us following recovery from one or more bleeds complicating portal hypertension. For the proposed portal decompression to be carried out the serum albumin had to be at least $3.0 \mathrm{~g} / 100 \mathrm{ml}$ and the serum bilirubin generally below $2-3 \mathrm{mg} / 100 \mathrm{ml}$. The patients operated on thus had an extremely good hepatic 'reserve'.

Perhaps the earliest thorough documentation of the occurrence of intrahepatic portal hypertension in the absence of cirrhosis was by McMichael in 1934. He described a portal inflammation or mild 'hepatitis' in the majority of his cases. Since this time sporadic reports have appeared describing similar conditions from various parts of the world (Imanaga et al, 1962; Ramalingaswami et al, 1962; Basu, Boyer,
Bhattacharya, Basu Mallik, and Sen Gupta, 1967; Tisdale et al, 1959; Mikkelsen et al, 1965). The geographical distribution may be apparent rather than real and may well be due to increasing awareness of the condition, with reclassification of existing cases previously misdiagnosed. Cer- $\overrightarrow{\vec{F}}$ tainly the majority of our cases had masqueraded $\widetilde{0}$ under the label of 'portal cirrhosis' although a 흘 number had been previously recognized as being $\frac{\bar{N}}{\bar{N}}$ 'fibrotic' rather than cirrhotic at operation (Hunt, $\overparen{\otimes}$ 1958). It would seem likely that further retrospective studies of other series will reveal more of these cases.

The present series of patients would seem $\overrightarrow{\vec{\omega}}$ most like those described by Mikkelsen et al $\stackrel{\omega}{\sigma}$ (1965), Basu et al (1967), and Nayak and Ramalingaswami (1969). A conspicuous difference, $\stackrel{.}{.}$ however, was the absence of intimal thickening of intrahepatic portal vein radicles in our biopsy or material. This thickening or sclerosis was con- $\vec{\circ}$ sidered by Mikkelsen and his coworkers (1965) 응 as the primary phenomenon affecting either the intrahepatic or the extrahepatic portal tree or $c$ both. This 'phlebosclerosis' seemed to us a consequence rather than a cause of the portal hyper- $\vec{\omega}$ tension, for only in the liver examined at necropsy was this change apparent, affecting larger intrahepatic portal radicles in four cases. However, similar intrahepatic portal vein sclerosis was also seen in a number of cirrhotic livers at necropsy. Nor could we find any comparable $\frac{\varnothing}{\varnothing}$ changes in liver architecture on review of $12 \propto$ wedge liver biopsies from patients with proven extrahepatic portal vein obstruction. In addition, 3 in this series the extrahepatic portal vein changes described, such as sclerosis and partial thrombosis, were found in both the non-cirrhotic and the cirrhotic patients. Evidence from other workers (Li, 1940; Hou and McFadzean, 1965) support 3 the view that these extrahepatic changes are $\dot{\delta}$ probably secondary to the portal hypertension. There was no doubt, however, that in all instances $ᄋ$ where follow-up biopsy or necropsy was obtained (Mikkelsen et al, 1965; Basu, Basu Mallik, Sen Gupta, Maddrey, Sen, Das, Biswas, and Pal, 1969; Nayak and Ramalingaswami, 1969), o including the present series, there was no pro- $N$ gression to cirrhosis. The lesion either remained static, or the fibrosis increased, or occasionally there was apparent improvement.

The majority of the portal venograms available for review showed some degree of distortion of $\stackrel{?}{?}$ intrahepatic pattern with an erratic lack of fine branches but no consistent abnormality could be made out. The apparent degree of distortion was often out of proportion to the histological findings. The 'cut-off' pattern described in the Indian patients (Basu et al, 1967) and incidentally also in the cases of 'tropical splenomegaly' (Leather, 1961; Williams et al, 1966) was not seen. The meaning of this abnormality, which comprised truncated main portal branches and lack of secondary and subsequent branches, was not 
clear. Talner, Boyer, and Clemett (1969) recorded four patients with presinusoidal portal hypertension who on venography had patent extrahepatic portal veins but occluded larger intrahepatic portal branches. In two cases these were shown to be due to multiple thrombi at necropsy; the origin of the thrombi was obscure. It would seem that more refined venographic techniques and injection-corrosion casts of future necropsy livers may clarify the situation.

We can offer no haemodynamic data as to the site of the portal bed block in our non-cirrhotic patients. We have, however, demonstrated, as did Boyer et al (1967), that in spite of such apparently minor histological changes, the height of the portal venous pressure was the same in noncirrhotic as in cirrhotic patients. Some workers (Polish et al, 1962; Imanaga et al, 1962; Mikkelsen et al, 1965) gave evidence for a presinusoidal block, whilst Basu et al (1967) demonstrated a raised wedged hepatic vein pressure with a normal or reduced liver blood flow, indicating a postsinusoidal obstruction. The possible mechanism for a presinusoidal elevation of portal pressure in these cases is a combination of an increased splenic vein inflow together with the portal tract lesions, but it is difficult to reconcile a postsinusoidal block with the absence of widespread regeneration nodules. It should be pointed out that four of our patients had undergone previous splenectomy, in two instances 13 and 17 years before bleeding varices were demonstrated, so in these cases at least the splenic inflow hypothesis is not appropriate.

The aetiology of the condition must remain speculative. In four of our cases there was unequivocal evidence of chronic arsenic poisoning, with death from lung cancer in two (Robson and Jelliffe, 1963). It is tempting to suggest that arsenic was also responsible for the liver lesion, and indeed Fowler's solution has been incriminated in the genesis of cirrhosis (Franklin, Bean, and Hardin, 1950), but the cases were poorly documented. Jaundice has also been associated with parenteral arsenic administration but this was presumably related to an associated serum hepatitis. Arsenic, however, cannot be incriminated in the majority of our cases. Changes subsequent upon multiple small intrahepatic portal vein thromboses are possible, but our material gives no supportive evidence for this. Long-continued increase in splenic venous inflow may produce secondary portal changes in the liver just as in the sequence Banti (1910) suggested, and the combination produces clinically significant portal hypertension. The stimulus for splenic enlargement then has to be explained, and in any case four of our patients had splenectomies many years before the appearance of portal hypertension. Unquestionably the liver lesion was static, when compared with hepatic cirrhosis, so one must postulate either that whatever factor or factors initiated the change are no longer acting, or that a factor such as is responsible for the perpetuation and progression of cirrhosis is absent. The possibility also exists that the condition may be a congenital hepatic anomaly, but the lack of family history and wide age range at presentation militate against this.

After portal decompression the complications $\bar{C}$ more usually associated with hepatic cirrhosis, 흘 apart from malignant changes, occurred to an $\frac{5}{7}$ equal extent in the non-cirrhotic group. Mik- $\stackrel{\varnothing}{\varnothing}$ kelsen et al (1965) and Basu et al (1967 and 1969) ڤ described significant encephalopathy occurring $\vec{\circ}$ in their patients but the incidence was less than the $18.2 \%$ found in the present series, where the incidence was in fact identical to the incidence of $O$ overt encephalopathy in the cirrhotic group. The occurrence of overt encephalopathy in the noncirrhotic groups was not surprising as this has been recorded in $15 \%$ of 47 patients with extra- ${ }^{\circ}$ hepatic portal hypertension (Thompson, Williams, $\vec{\circ}$ and Sherlock, 1965), and in a patient with a 윽 normal liver subjected to portal systemic anastomosis (McDermott and Adams, 1954). The $\subseteq$ encephalopathy in our group, as in the extrahepatic cases of Thompson et al (1965), may have $\vec{\theta}$ been related to the observed sequential deteriora- ? tion in liver function associated with diversion of portal blood from the liver and with advancing age. In spite of this deterioration survival was prolonged and death from liver failure was relatively uncommon, only four of 13 patients dying from this cause. Basu et al (1969) reported deaths remote from operation from liver failure in one of three patients and Mikkelsen et al (1965) deaths from liver failure in five of six patients. There was no correlation, at least in our cases, between age the extent of deterioration of liver function and death from liver failure. Only two of these patients came to necropsy and in one the liver was irregular and shrunken with an increase in fibrous tissue.

The superior survival time of the non-cirrhotic $\frac{\text { 의 }}{3}$ group when compared with that of the cirrhotic $D$ group (Fig. 5) has been recognized in other series. Mikkelsen et al (1965) and Basu et al (1969) N report good prognosis following portal decompression; the former authors reported postoperative survivals of from two to 15 years in 14 of 23 of their cases with patent and partially patent portal veins. It would therefore be in- 6 valuable to be able to differentiate these patients before surgery as they are clearly the ideal candidates for portal decompression. The present series, however, draws attention to the difficulties involved in doing this, and it would appear in the individual case impossible to distinguish the condition from hepatic cirrhosis either clinically or biochemically. Definitive diagnosis, therefore, rested upon the venographic findings, the macroscopic appearance of the liver, and the absence of cirrhosis on wedge biopsy. Nayak and Ramalingaswami (1969) advocated a deep needle biopsy in addition as occasional discrete nodula- 


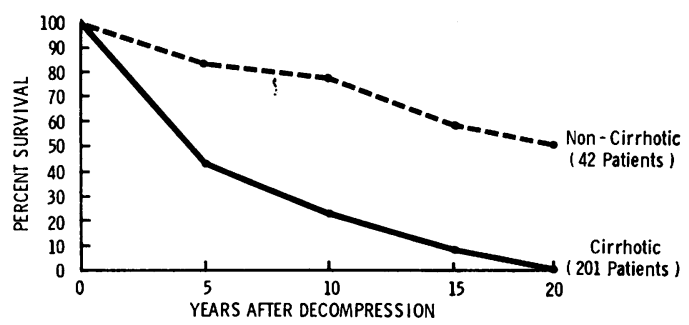

Fig. 5 Cumulative postoperative survival of patients with intrahepatic portal hypertension.

tion and more severe fibrosis may exist near the liver surface and be confusing.

The far better prognosis of the non-cirrhotic group serves to underline the validity of the histological findings and their remarkable lack of progression.

This work was supported by the Endowment Fund of the Board of Governors of St Bartholomew's Hospital.

\section{References}

Banti, G. (1910). Uber Morbus Banti. Folia haemat. (Lpz.), 10, 33-74.

Basu, A. K., Basu Mallik, K. C., Sen Gupta, K. P., Maddrey, W. C., Sen, N. N., Das, M. M., Biswas, S. K., and Pal, N. C. (1969). Long term follow up studies of non-cirrhotic portal fibrosis after surgery. Brit. J. Surg., 56, 497-503.

Basu, A. K., Boyer, J., Bhattacharya, R., Basu Mallik, K. C., and Sen Gupta, K. P. (1967). Non-cirrhotic portal fibrosis with portal hypertension, a new syndrome. Part I. Clinical and function studies and results of operations. Ind.J. med. Res., $55,336-350$.

Basu Mallik, K. C., Sen Gupta, K. P., Basu, A. K., Biswas, S. K., Pal, N. C., and Boyer, J. (1967). Non-cirrhotic portal fibrosis with portal hypertension. A new syndrome. Part II Histopathological studies. Ind. J. med. Res., 55, 351-359.

Boyer, J. L., Sen Gupta, K. P., Biswas, S. K., Pal, N. C., Basu Mallik, K. C., Iber, F. L., and Basu, A. K. (1967). Idiopathic portal hypertension. Comparison with the portal hypertension of cirrhosis and extrahepatic portal vein obstruction. Ann. intern. Med., 66, 41-68.

Cohn, R., and Blaisdell, F. W. (1968). The natural history of the patient with cirrhosis of the liver with esophageal varices following the first massive hemorrhage. Surg. Gynec. Obstet., 106, 699-701.

Crile, G., Jr. (1953). Treatment of oesophageal varices by transoesophageal obliteration. Surg. Gynec. Obstet., 96, 573-576.

Franklin, M., Bean, W. B., and Hardin, R. C. (1950). Fowler's solution as an etiologic agent in cirrhosis. Amer. J. med. Sci., n.s., 219, 589-596.

Hou, P. C., and McFadzean, A. J. S. (1965). Thrombosis and intimal thickening in the portal system in cirrhosis of the liver. J. Path. Bact., 89, 473-480.

Hunt, A. H. (1958). A Contribution to the Study of Portal Hypertension. Livingstone, Edinburgh.
Imanaga, H., Yamamoto, S., and Kuroyanagi, Y. (1962). Surgical treatment of portal hypertension according to state of intrahepatic circulation. Ann. Surg., 155, 42-50.

Kerr, D. N. S., Harrison, C. V., Sherlock, S., and Walker, R. M. (1961). Congenital hepatic fibrosis. Quart. J. Med., 30, 91-117.

Leather, H. M. (1961). Portal hypertension and gross splenomegaly in Uganda. Brit. med. J., 1, 15-18.

Li, P. L. (1950). Adaptation in veins to increased intravenous pressure, with special reference to the portal system and inferior vena cava. J. Path. Bact., 50, 121-136.

McDermott, W. V., and Adams, R. D. (1954). Episodic stupor associated with an Eck fistula in the human with particular reference to the metabolism of ammonia. J. clin. Invest., $33,1-9$.

McMichael, J. (1934). The pathology of hepato-renal fibrosis. J. Path. Bact., 39, 481-502.

Mikkelsen,W. P., Edmonson, H. A., Peters, R. L., Redeker, A. G., and Reynolds, T. B. (1965). Extra and intrahepatic portal hypertension without cirrhosis (hepato-portal sclerosis). Ann. Surg., 162, 602-620.

Miller, M. C., and Brandt, J. L. (1962). Portal hypertension in the absence of both liver disease and vascular obstruction. Amer. J. dig. Dis., 7, 442-448.

Nayak, N. C., and Ramalingaswami, V. (1969), Obliterative portal venopathy of the liver. Arch. Path., 87, 359-369.

Polish, E., Christie, J., Cohen, A., and Sullivan, B., Jr. (1962). Idiopathic presinusoidal portal hypertension (Banti's syndrome). Ann. intern. Med., 56, 624-627.

Porter, G. H. (1961). Hepatic sarcoidosis-a cause of portal hypertension and liver failure. Arch. intern. Med., 108, $482-495$.

Ramalingaswami, V., Wig, K. L., and Sama, S. K. (1962). Cirrhosis of the liver in Northern India: a clinico-pathological study. Arch. intern. Med., 110, 350-358.

Reynolds, T. B., Hidemura, R., Michel, H., and Peters, R. (1969). Portal hypertension without cirrhosis in alcoholic liver disease. Ann. intern. Med., 70, 497-506.

Robson, A. O., and Jelliffe, A. M. (1963). Medicinal arsenic poisoning and lung cancer. Brit. med. J., 2, 207-209.

Shaldon, S., and Sherlock, S. (1962). Portal hypertension in the myeloproliferative syndrome and the reticuloses. Amer. $J$. Med., 32, 758-764.

Sherlock, S. (1964). Haematemesis in portal hypertension. Brit. J. Surg., 51, 746-749.

Sherlock, S., Feldman, C. A., Moran, B., and Scheuer, P. J. (1966) Partial nodular transformation of the liver with portal hypertension. Amer. J. Med., 40, 195-203.

Siderys, H, and Vellios, F, (1964). Portal hypertension without cirrhosis or extrahepatic obstruction. Report of a case. Amer. J. Surg., 108, 785-789.

Stansfeld, A. G., and Zeegen, R. (In preparation.) Pathological studies in non-cirrhotic portal hypertension.

Stone, W. D., Islam, N. R. K., and Paton, A. (1968). The natural history of cirrhosis. Quart. J. Med., 37, 119-132.

Talner, L. B., Boyer, J. L., and Clemett, A. R. (1969). Intrahepatic portal vein occlusion: a cause of portal hypertension. Radiology, 92, 1265-1270.

Thompson, E. N., Williams, R., and Sherlock, S. (1964). Liver function in extrahepatic portal hypertension. Lancet, 2, $1352-1356$.

Tisdale, W. A., Klatskin, G., and Glenn, W. W. L. (1959). Portal I hypertension and bleeding oesophageal varices. Their occurrence in the absence of both intrahepatic and extrahepatic obstruction of the portal vein. New Engl. J. Med., 261, 209-218.

Williams, R., Parsonson, A., Somers, K., and Hamilton, P. J. S. N (1966). Portal hypertension in idiopathic tropical splenomegaly. Lancet, 1, 329-333.

Zeegen, R., Drinkwater, J. E., and Dawson, A. M. (1970). A method for measuring cerebral dysfunction in patients with liver disease. Brit. med. J., 2, 633-636.

Zeegen, R. Drinkwater, J. E., Fenton, J. C. B., Vince, A., and Dawson, A. M. (1970). Some observations on the effects of treatment with lactulose in patients with chronic hepatic encephalopathy. Quart. J. med., 39, 245-263.

Zeegen, R., Stansfeld, A. G., Dawson, A. M., and Hunt, A. H. H. (1969). Bleeding oesophageal varices as the presenting feature in primary biliary cirrhosis. Lancet, 2, 9-13. 